\title{
Electrospun synthesis of silver/poly (vinyl alcohol) nano-fibers: investigation antibacterial activity and flame retardant property
}

\author{
Sara Azarakhsh \\ Hamed Bahiraei ( $\nabla$ hamedbahiraei@gmail.com ) \\ https://orcid.org/0000-0002-9641-5889 \\ Gholamhosain Haidari \\ Davood Ghanbar
}

\section{Research Article}

Keywords: Nano-fibers, Nanocomposites, Electro-spinning, Antibacterial

Posted Date: February 26th, 2021

DOI: https://doi.org/10.21203/rs.3.rs-248410/v1

License: (c) (i) This work is licensed under a Creative Commons Attribution 4.0 International License.

Read Full License 


\section{Abstract}

Polyvinyl alcohol (PVA) and Ag nanoparticles-embedded polyvinyl alcohol (Ag/PVA) nano-fibers have been synthesized successfully via the electro-spinning technique at room temperature. XRD patterns confirmed the presence of Ag nanoparticles in the electro-spinning PVA nano-fibers. FE-SEM images displayed that silver nanoparticles with an average particle size of $32 \mathrm{~nm}$ are uniformly dispersed in PVA nano-fibers. Also, the average diameter of Ag/PVA nano-fibers was estimated to be $142 \mathrm{~nm}$. The EDX analysis of Ag/PVA nano-fiber determined the aggregation of Ag nanoparticles in the range of polymer nano-fibers. The antibacterial property of Ag/PVA nano-fiber for inhibition of Escherichia coli (E-coli) growth was approved. Since polyvinyl alcohol is a biocompatible and water-soluble polymer, it can be considered as unique candidate for the preparation of wound dressing nano-fibers. It is also found that the nontoxic nanostructures can appropriately enhance both flame retardant and thermal stability properties of the Ag/PVA matrix. These results show that the Ag/PVA nano-fibers may find practical applications as self-reinforced medical devices and tissue-engineering scaffolds.

\section{Introduction}

The nanostructured materials with their unique properties can be modified and controlled in optical properties and surface functionalization [1]. They have been applied in various fields of application like energy, health, medicine, biotechnology, electronics, environmental food, and agriculture. Transition metal nanoparticles, such as zinc ( $\mathrm{Zn})$, iron ( $\mathrm{Fe})$, gold $(\mathrm{Au})$, and silver $(\mathrm{Ag})$, have great opportunities in biomedical applications because of the low volume ratio to surface area [2-3]. Among such transition metal elements, Ag is the most attractive because of its low human toxicity, wide spectrum, and strong activity. Recently, the inducement of metal nanoparticles on certain surfaces to form macroscopic 3D structures has attracted a great deal of attention, which can be used in catalysis, cancer treatment, optical sensing, and electronic devices [4]. The electrospun method is one of the simple and effective techniques that under a strong electrostatic field can extract continuous nano-fibers from melts or polymer solutions [5]. This technique has received special attention for healthcare textiles and medicine due to its effectiveness in producing fibrous structures or nano-fibers. By adjusting the manufacturing parameters and polymer solution types, the fibers can be produced with various diameters from few nanometres to several micrometres. Each type of nano-fiber that prepared from various polymers, like synthetic and natural polymers, has several attractive characteristics like the flexibility of the surface, functionality, high porosity, and specific surface area [6]. The produced nano-fibers can be applied as photoelectric devices, components of biosensors, wound dressings, tissue scaffolds, filter materials, biomedical elements, vascular catalysts, and reinforced composite materials [7]. Polyvinyl alcohol (PVA) is a synthetic and nontoxic polymer that is known as a water-soluble polymer. Due to its excellent biocompatibility, chemical resistance, biodegradability, and good fiber-forming property, it is widely used in practical applications [8]. Ultrafine Polyvinyl alcohol fibers with different potential applications can be prepared by electrospun process. The electrospun of hybrid materials consisting of polymers and metal 
nanoparticles within the nanofibrous materials has become one of the most promising and growing technologies [9].

Recently, the utilization of functional nanoparticles in the PVA nano-fiber has been widely applied because of their uniquely promising properties. The Ag/PVA nano-fibers can show much anti-microbial efficiency than conventional Ag/PVA micro-fibers due to their low volume ratio to surface area. Many research papers have investigated the antibacterial properties of Ag nanoparticles [10-12]. For medical applications, PVA nano-fiber with metal nanostructure is more active than the large-scale modification of material [13]. This research focused on the characterization and antibacterial study of PVA/Ag nanofibers produced by the electro-spinning technique that is a promising candidate for applications as wound dressings.

\section{Experimental}

\subsection{Materials and Characterization}

All reagents, including PVA, $\mathrm{Ag}\left(\mathrm{NO}_{3}\right)_{2}, \mathrm{NaBH}_{4}$, and starch were the analytical grade and used without further purification. The phase formation of the prepared nano-fibers was identified using X-ray diffraction (XRD, $\lambda=1.5418 \AA$ ). Field-emission scanning electron microscopy (FE-SEM) was used to study the morphology of samples. FTIR spectra were taken on an AVATAR 360 Fourier transform infrared spectrometer. The thermal stability of the prepared nanocomposite was studied by thermogravimetric analysis(TGA).

\section{2 preparation of Ag nanoparticles}

$0.10 \mathrm{~g}$ of $\mathrm{AgNO}_{3}$ in distilled water $(100 \mathrm{ml})$ was dissolved. Then, $0.5 \mathrm{~g}$ starch as a surfactant was added to the previous solution. About $2 \mathrm{ml}$ of $\mathrm{NaBH}_{4}$ solution ( $10 \mathrm{~g} / \mathrm{lit}$ ) was slowly mixed to the above solution, and the solution was stirred for $15 \mathrm{~min}$. After that, the prepared precipitation was collected by centrifugation, washed, and dried at room temperature.

\subsection{Synthesis of PVA nano-fibers}

Various concentrations (5-15\%) of polyvinyl alcohol and distilled water were prepared as the starting solutions. The solution was placed on a magnetic stirrer and set to $50^{\circ}$ for 5 hours. The solution was well dissolved and ready to spin.

\subsection{Synthesis of (90\%:10\%) Ag/PVA nano-fibers}

Using the ratio of $10 \%$ silver nanoparticles synthesized to the polymer solution, add $0.1 \mathrm{~g}$ of silver nanoparticles powder to $0.9 \mathrm{~g}$ of polyvinyl alcohol solution and place at $50^{\circ} \mathrm{C}$ on the magnetic stirrer for $3 \mathrm{~h}$ to obtain a uniformly concentrated solution. Then put in an ultrasonic bath for 20 minutes and then ready to spin. 


\section{Results And Discussion}

Fig.1 displays a schematic diagram of the experimental setup (precipitation procedure) that was used for nanoparticle preparation. Fig.2 shows the schematic of the electro-spinning process for producing nanofibers with a high voltage around 22kV, distance between needle and collector $150 \mathrm{~mm}$, and flow rate about $0.5 \mathrm{mlh}^{-1}$. To determine the phase structure of the prepared samples, the X-ray diffraction analysis was performed at room temperature, over the $2 \theta$ angular range $10-80$ 。. Fig. 3 shows the XRD pattern of the electrospun PVA nano-fibers. There are two broad hump peaks at $2 \theta=20$ and 51 indexed as (101) and (200) planes attributed to the semi-crystallinity nature of the electrospun PVA nano-fibers. These peaks may be related to the intermolecular hydrogen bonding between PVA molecular chains [14]. The XRD pattern of Ag/PVA nano-fibers, is shown in Fig.4. There are extra diffraction peaks at $2 \theta=38.1^{\circ}$ and $77.4^{\circ}$ that can be assigned to the ( $\left.\begin{array}{lll}1 & 1 & 1\end{array}\right)$ and ( $\left.\begin{array}{lll}3 & 1 & 1\end{array}\right)$ planes of FCC structure of Ag element (JCPDS; \# 04-0783), respectively. It is seen that for Ag/PVA fibers the peak intensity at $2 \theta=20$ decreased because of the complexation of Ag nanoparticles in PVA nano-fibers.

Fig .5 shows the morphology of $15 \%$ concentration of PVA nano-fibers. The presence of many PVA noodles shows the formation of an inhomogeneous structure during the electrospun process. It is seen that there are no noticeable nano-fibers in this sample. The SEM images of PVA nano-fibers that were prepared of $10 \%$ concentration are shown in Fig.6. It can be seen the uniform and suitable mono-disperse nano-fibers with average diameter size less than $336 \mathrm{~nm}$ successfully are prepared. The SEM images of the silver nanoparticles, prepared using starch as a capping and surface-active agent, is illustrated in Fig.7. This figure clearly shows the Ag nanoparticles with spherical shape, and the average particles size of about $32 \mathrm{~nm}$. Fig. 8 displays the SEM images of Ag/PVA composite nano-fibers. These images confirm that the nano-fibers with an average diameter size of $142 \mathrm{~nm}$ are synthesized. The presence of silver nanoparticles in the polymeric fiber matrixes is confirmed. Also, for better identification, the energy dispersive X-ray (EDX) analysis of PVA-Ag nano-fibers was taken, and the result is shown in Fig.9. The EDX spectrum approved the presence of all three elements silver, carbon, and oxygen with $\mathrm{K}_{\mathrm{a}}$ of carbon and oxygen and also $L_{a}$ and $L_{\beta}$ of silver, respectively.

Fig.10 illustrates the FT-IR spectrum of the prepared PVA nano-fibers. It can be seen that the absorption peaks of pure PVA are at 3585, 2976, 1742, 1571, 1461 and $846 \mathrm{~cm}^{-1}$ [15]. Also, there is a broad and strong absorption band between $3400-3200 \mathrm{~cm}^{-1}$, that is attributed to the vibration stretching in the hydroxyl (O-H) group. The obtained band at $2921 \mathrm{~cm}^{-1}$ is a result of the $\mathrm{C}-\mathrm{H}$ stretching vibration. The absorption bands at 1740 and $1570 \mathrm{~cm}^{-1}$ are due to stretching vibration in $\mathrm{C}=0$. The presence of band at 1140 and $910 \mathrm{~cm}^{-1}$ are attributed to stretching vibrations in $\mathrm{C}-0$, while the band at $838 \mathrm{~cm}^{-1}$ is attributed to stretching vibration in $\mathrm{C}-\mathrm{C}[16]$. Fig.11 displays the FT-IR spectrum of the Ag/PVA nano-fibers. This spectrum shows the broad absorption peak at 2939 and $3348 \mathrm{~cm}^{-1}$ corresponding to the stretching mode of $\mathrm{C}-\mathrm{H}$ group and the $\mathrm{O}-\mathrm{H}$ group of the hydroxyl group, respectively. Absorption bands at 1033 and 1730 $\mathrm{cm}^{-1}$ are assigned to the stretching mode of $\mathrm{C}-\mathrm{O}$ and $\mathrm{C}=\mathrm{O}$ bonds, respectively. The obtained broadening and the little decreasing intensity in the absorption bands range around 3300, 2970, 1740, $1460 \mathrm{~cm}^{-1}$ and 
vanishing of bands at 1560,1630 , and $1380 \mathrm{~cm}^{-1}$ obviously propose the interaction that occurred between Ag nanoparticles and PVA nano-fibers.

Fig.12 shows the antibacterial efficacy of Ag/PVA nano-fiber against E.Coli. For better comparison, both Gentamicin disc and control disk were inserted beside synthesized Ag/PVA disc. As we expected, there is no inhibitor zone around the control disc while the diameter around Gentamicin disc treatment is about 4 $\mathrm{cm}$. The diameter of the growth inhibitor zone in the treatment of the desired material is around $2 \mathrm{~cm}$.

Thermal gravimetric analysis (TGA) of the Ag/PVA nanocomposite is depicted in Fig. 13. It is found that with the addition of Ag nanoparticles to the polyvinyl alcohol, the thermal stability of Ag/PVA nano-fibers was improved. As the nanostructured materials can act as dams, which slow the production of thermal transport and evaporation during polymer decomposition, the existence of nanoparticles can shift the thermal decomposition towards higher temperatures. Silver effect on the fire retardancy of the cellulose acetate and has been examined applying UL-94 test (sample $130 \times 13 \times 1.6 \mathrm{~mm}$ is applied). A fire (1.5 $\mathrm{cm}$ ) is used to sample (time: $10 \mathrm{~s}$ ) twice. When the specimen is extinguished in less than 10 seconds after fire contact classified as $\mathrm{V}-0$, particle drips are allowed as long as they are not inflamed. A V- 1 type is for a sample with fire time less than $30 \mathrm{~s}$ (drips are like V-0). V-2 classification has fire time like V-1 while flaming drips are permitted. When the total flaming time is above $50 \mathrm{~s}$ it is not classified (NC); finally, horizontal burning with a rate less than $76 \mathrm{~mm} / \mathrm{min}$ is HB [17].

UL-94 tests for pure PVA is V-2 while Ag/PVA approves V-0 classification (Fig 14). Flame retardancy of nanocomposite is because of high surface to volume ratio, which can disperse into the matrix homogeneously, and formation of a char dam during the combustion. This dam decreases the evaporation of organic segments and reduces oxygen and flame, reaching the product.

\section{Conclusions}

Ag nanoparticles were prepared by an easily applicable chemical reaction at room temperature. Polyvinyl alcohol nano-fibers and their nanocomposites with Ag were prepared by the electro-spinning method. The FE-SEM images showed that silver nanoparticles are uniformly dispersed in PVA nano-fibers. The average diameter of Ag/PVA nano-fibers was about $142 \mathrm{~nm}$. Silver antibacterial property for inhibition of E-Coli growth was investigated. Also, thermal stability and flame retardant property of the Ag/PVA matrix were studied. Results showed that the Ag/PVA nano-fibers may find practical applications as self-reinforced medical devices, tissue-engineering scaffolds and flame retardant material.

\section{Declarations}

We declare that we have no financial and personal relationships with other people or organizations that could have appeared to influence the work reported in this paper.

\section{References}


[1] Ibrahim Khan, Khalid Saeed, Idrees Khan, Nanoparticles: Properties, applications and toxicities, Arabian Journal of Chemistry, 12 (2019) 908-931.

[2] Shyam Baboo Prasad, Vidhu Aeri, Yashwant, Current Understanding of Synthesis and Pharmacological Aspects of Silver Nanoparticles, American Journal of Phytomedicine and Clinical Therapeutics, 1 (2013) 536-547.

[3] Rahmawati R, Melati A, Taufiq A, Sunaryono, Diantoro M, Yuliarto B, Suyatman S, Nugraha N and Kurniadi D, Preparation of MWCNT-Fe304 Nanocomposites from Iron Sand Using Sonochemical Route,IOP Conference Series: Materials Science and Engineering , 202 (2017) 012013.

[4] Rafael Contreras Caceres, Claudine Dawson, Petr Formanek, Dieter Fischer, Frank Simon, Andreas Janke, Petra Uhlmann and Manfred Stamm , Polymers as Templates for Au and Au@Ag Bimetallic Nanorods:UV-Vis and Surface Enhanced Raman Spectroscopy, Chemistry of Materials, 25 (2013) 158169.

[5] Chang-Mou Wu, Hau-Geng Chiou, Siang-Ling Lin, Jian-Min Lin, Effects of electrostatic polarity and the types of electrical charging on electrospinning behaviour, Journal of Applied Polymer Science,126 (2012) 89-97.

[6] Tatang Wahyudi, Doni Sugiyana, Pembuatan Serat Nano Menggunakan Metode Electrospining, Arena Tekst 26 (2011) 29-34.

[7] Milena Ignatova,Kirilka Starbova,Nadya Markova,Nevena Manolova and lliya Rashkov, Electrospun nano-fibre mats with antibacterial properties from quaternised chitosan and poly(vinyl alcohol), Carbohydrate Research, 341 (2006) 2098-2107.

[8] Chun Ho Kim, Kyu Suk Choi, Synthesis and Antibacterial Activity of Quaternized chitosan Derivatives Having Different Methylene Spacers, Journal of Industrial and Engineering Chemistry, 8 (2002) 71-76.

[9] Zhijie Zhang, Yunping Wu, Zhihua Wang , Xueyan Zou, Yanbao Zhao, Lei Sun, Fabrication of silver nanoparticles embedded into polyvinyl alcohol (Ag/PVA) composite nanofibrous films through electrospinning for antibacterial and surface-enhanced Raman scattering (SERS) activities, Materials science and Engineering, 69 (2016) 462-469.

[10] Lin Li, Yi Li, Jiashen Li, Lei Yao, Arthur F. T. Mak, Frank Ko, Ling Qin, Antibacterial Properties of Nanosilver PLLA FibrousMembranes, Journal of Nanomaterials , 209 (2009) 168041.

[11] Mahenda Rai ,Alka Yadav ,Aniket Gade, Silver nanoparticles as a new generation of antimicrobials, Biotechnology Advances , 27 (2009) 76-83.

[12] O. Akhavan, Lasting antibacterial activities of $\mathrm{Ag}-\mathrm{TiO} 2 / \mathrm{Ag} / \mathrm{a}-\mathrm{TiO} 2$ nanocomposite thin film photocatalysts under solar light irradiation, Journal of Colloid and Interface Science , 336 (2009) 117124. 
[13] D. R. Paul ,L. M. Robeson, Polymer nanotechnology: Nanocomposites, Polymer, 49 (2008) 3187-3204.

[14] Shahla Ahmadian Fard Fini , Masoud Salavati Niasari , Davood Ghanbari, Hydrothermal green synthesis of magnetic Fe304-carbon dots by lemon and grape fruit extracts and as a photoluminescence sensor for detecting of E.coli bacteria, Spectrochimica Acta Part A: Molecular and Biomolecular, 203 (2018) 481-493.

[15] Shahla Ahmadian-Fard-Finia, Davood Ghanbari, Omid Amiri, Masoud Salavati-Niasari, Electrospinning of cellulose acetate nano-fibers/Fe/carbon dot as

photoluminescence sensor for mercury (II) and lead (II) ions, Carbohydrate Polymers, 229 (2020) 115428.

[16] Banafsheh Moradi , Gholamreza Nabiyouni, Davood Ghanbari , Rapid photo-degradation of toxic dye pollutants: green synthesis of mono-disperse $\mathrm{Fe} 3 \mathrm{O} 4-\mathrm{CeO} 2$ nanocomposites in the presence of lemon extract, Journal of Materials Science: Materials in Electronics, 29 (2018) 11065-11080.

[17] Shahla Ahmadian Fard Fini , Davood Ghanbari, Masoud Salavati-Niasari, Photoluminescence carbon dot as a sensor for detecting of Pseudomonas aeruginosa bacteria: Hydrothermal synthesis of magnetic hollow NiFe204-carbon dots nanocomposite material , Composites Part B: Engineering ,161 (2019) 564577.

\section{Figures}


Stage 1

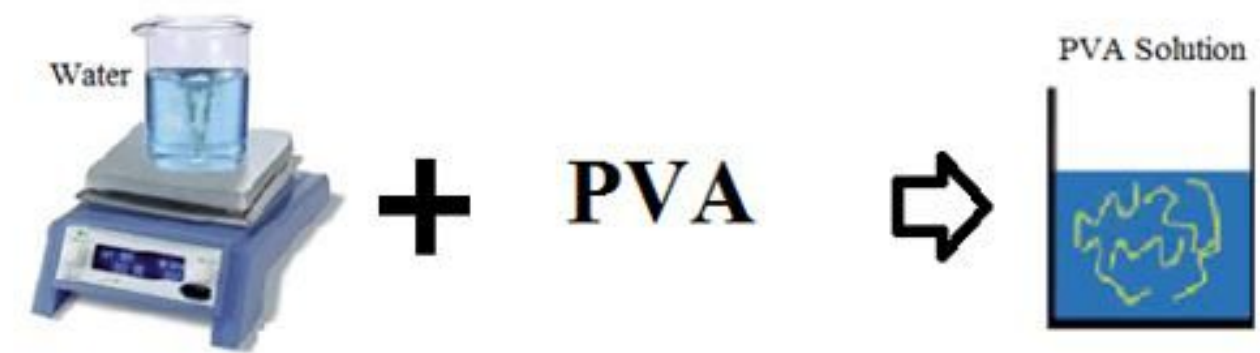

Stage 2

Silver Nanoparticles(Ag)

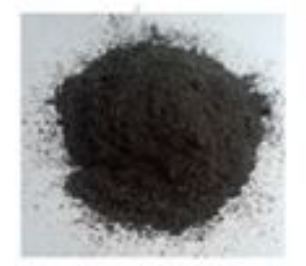

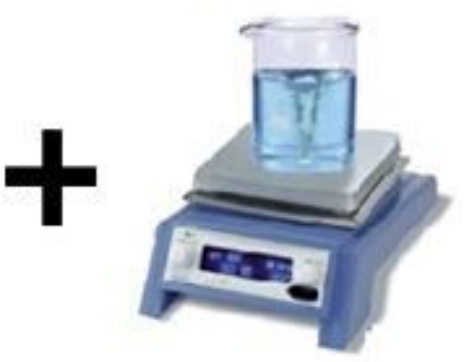

PVA Solution

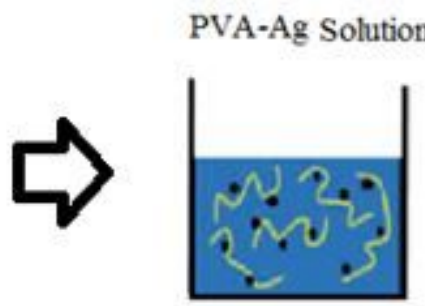

PVA-Ag Nanotibers
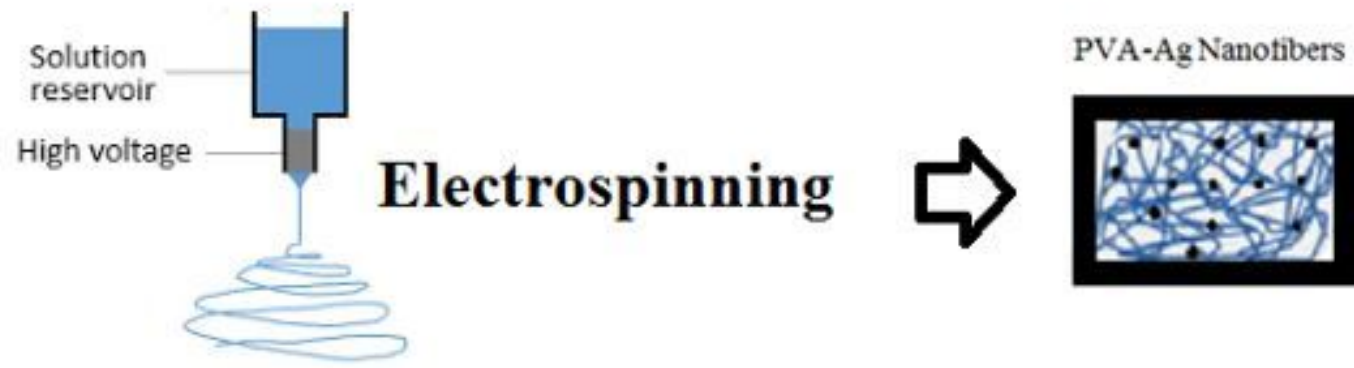

Figure 1

The schematic of the experimental procedure for synthesizing Ag/PVA nano-fibers 


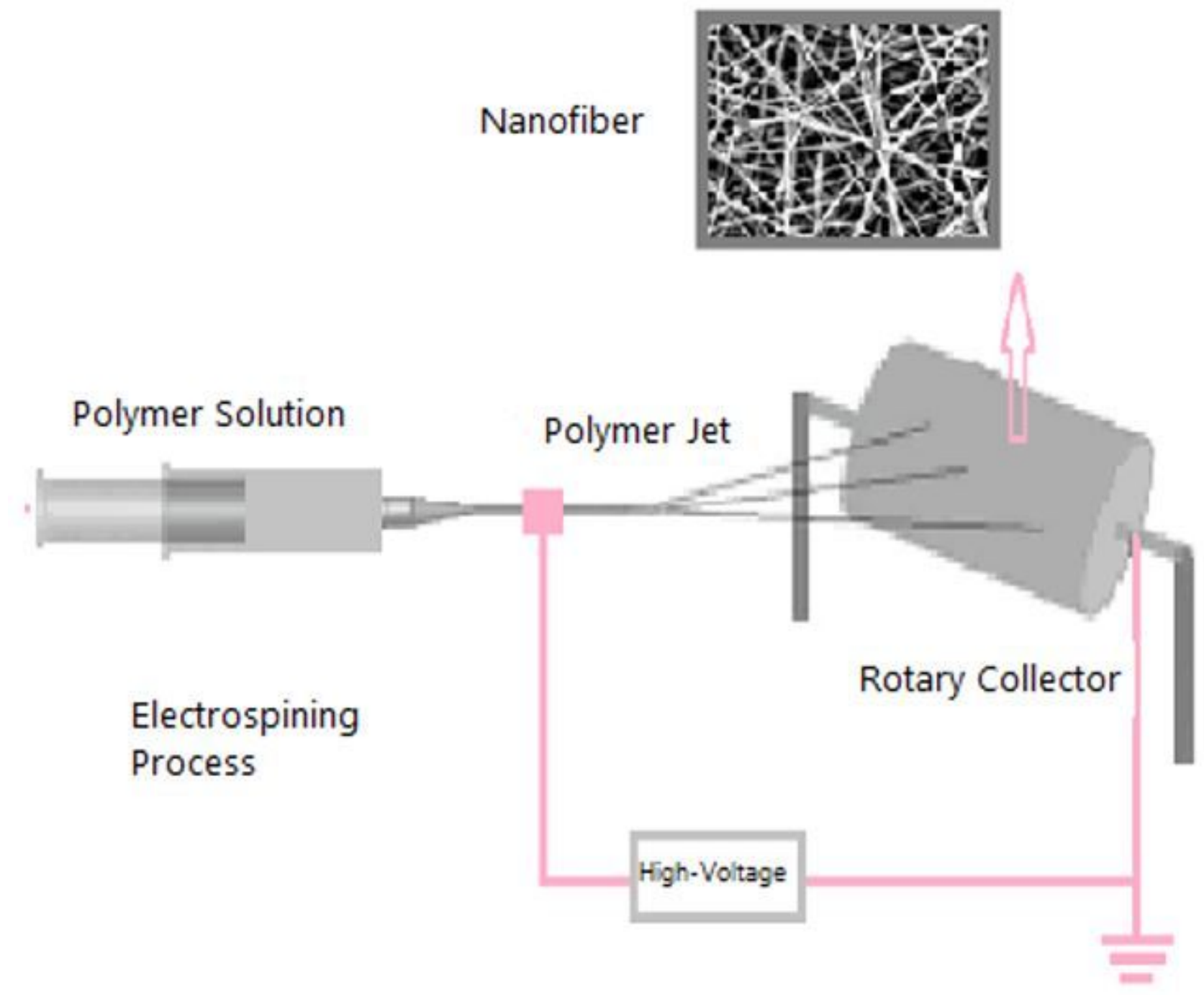

Figure 2

Schematic of Electro-spinning process 


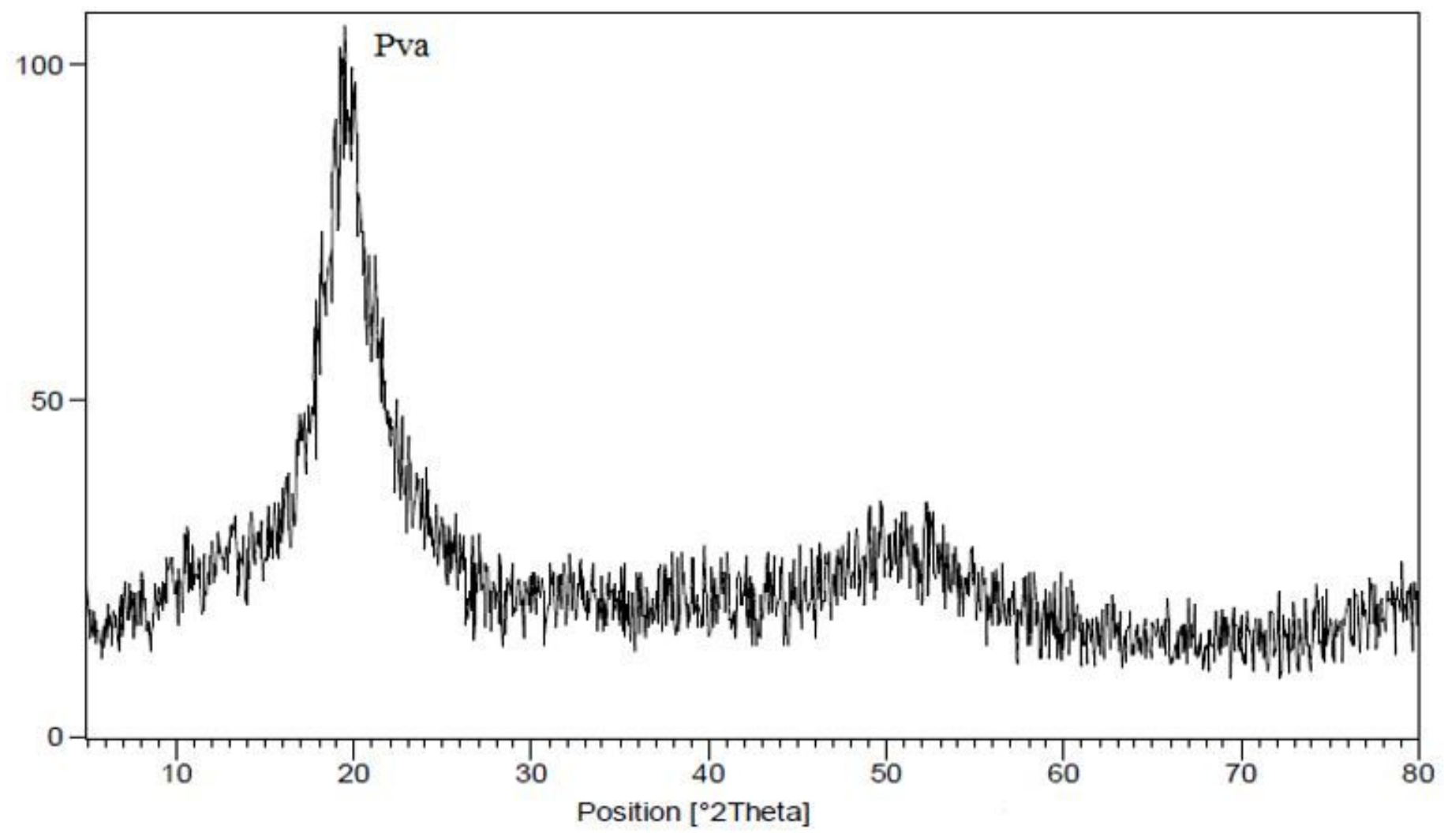

Figure 3

XRD pattern of PVA nano-fibers 


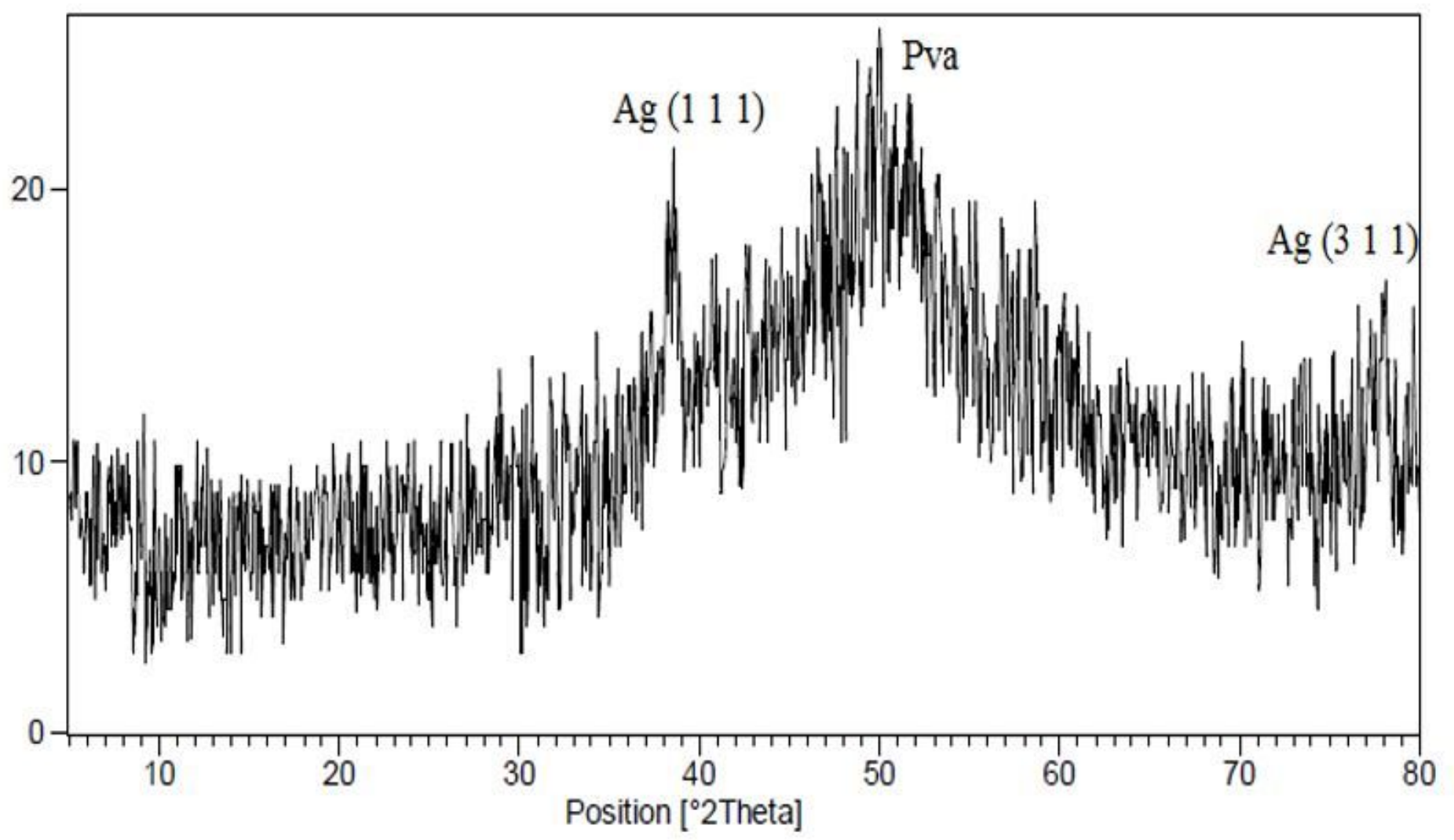

Figure 4

XRD pattern of Ag/PVA nano-fibers 


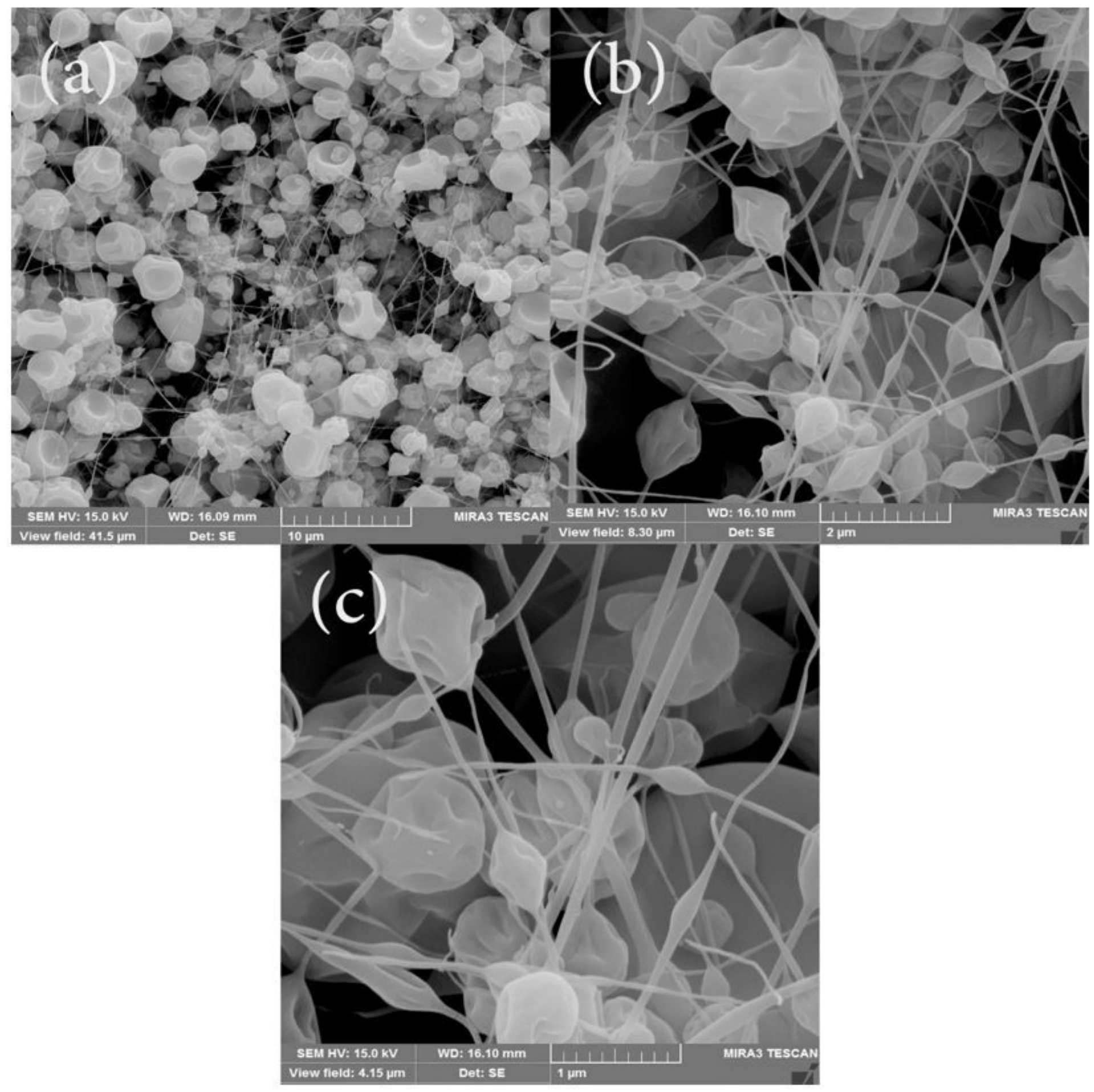

Figure 5

SEM images of PVA nano-fiber obtained from $15 \%$ concentration solution 


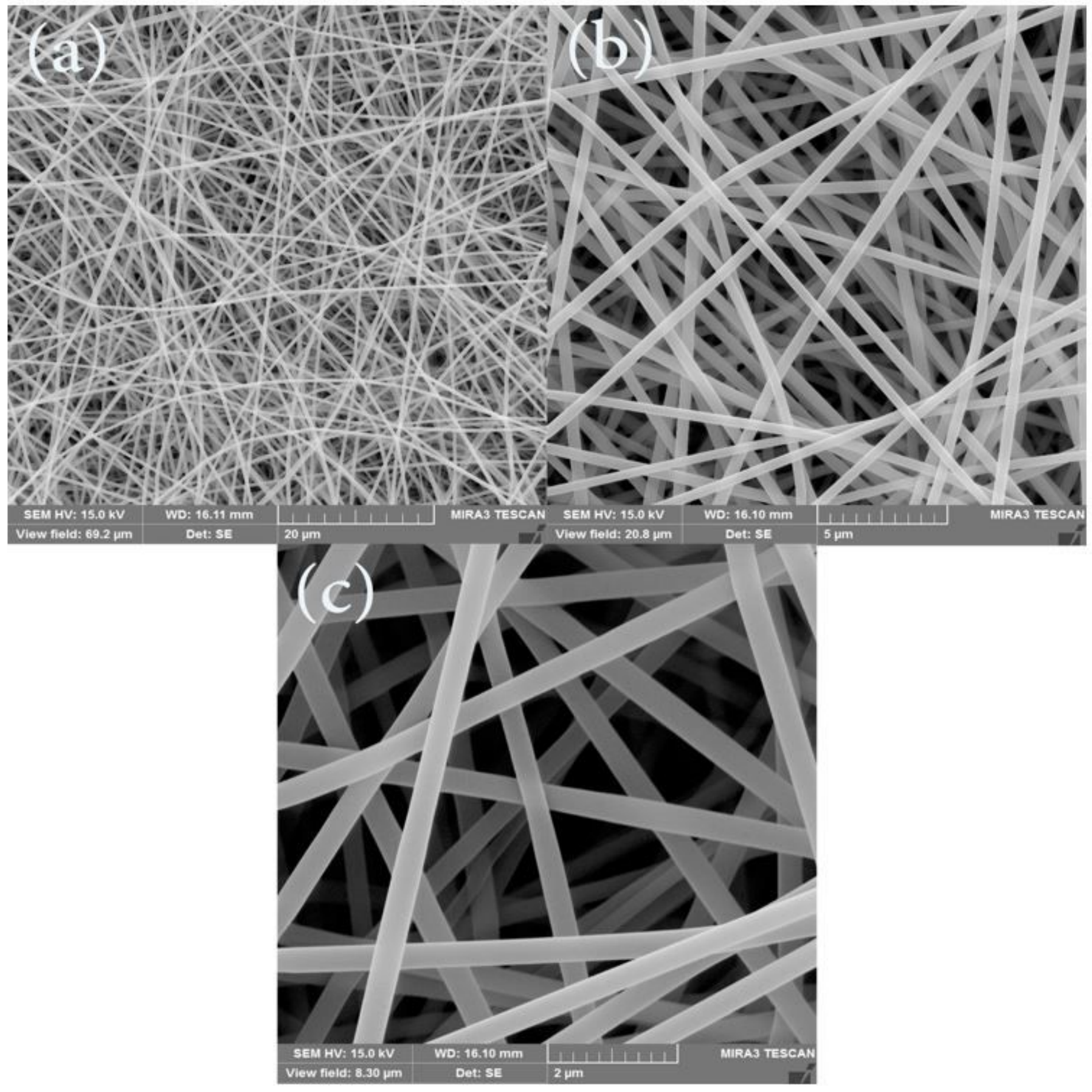

Figure 6

SEM images of PVA obtained from $10 \%$ concentration solution 


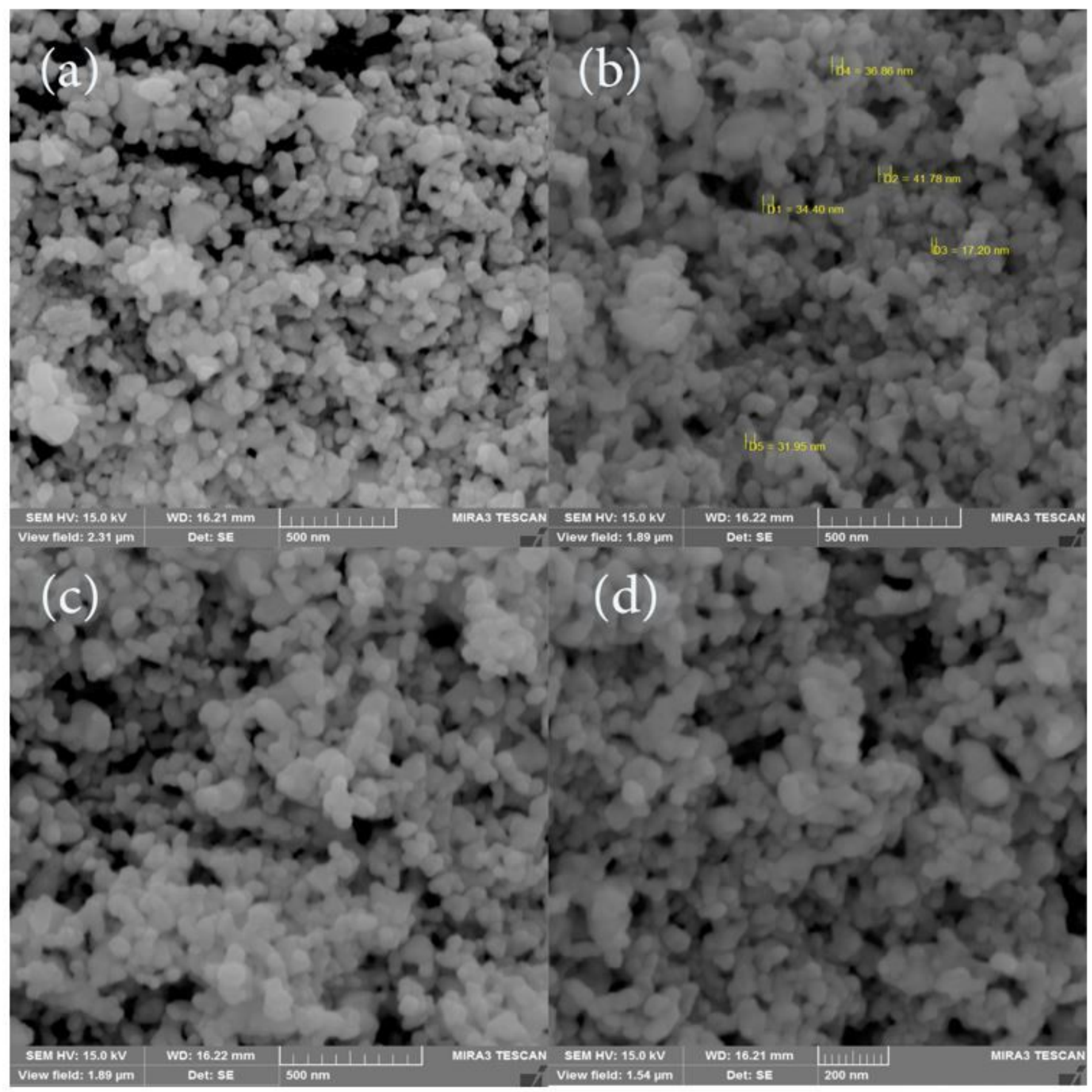

\section{Figure 7}

SEM images of Ag nanoparticles prepared in the presence of starch 


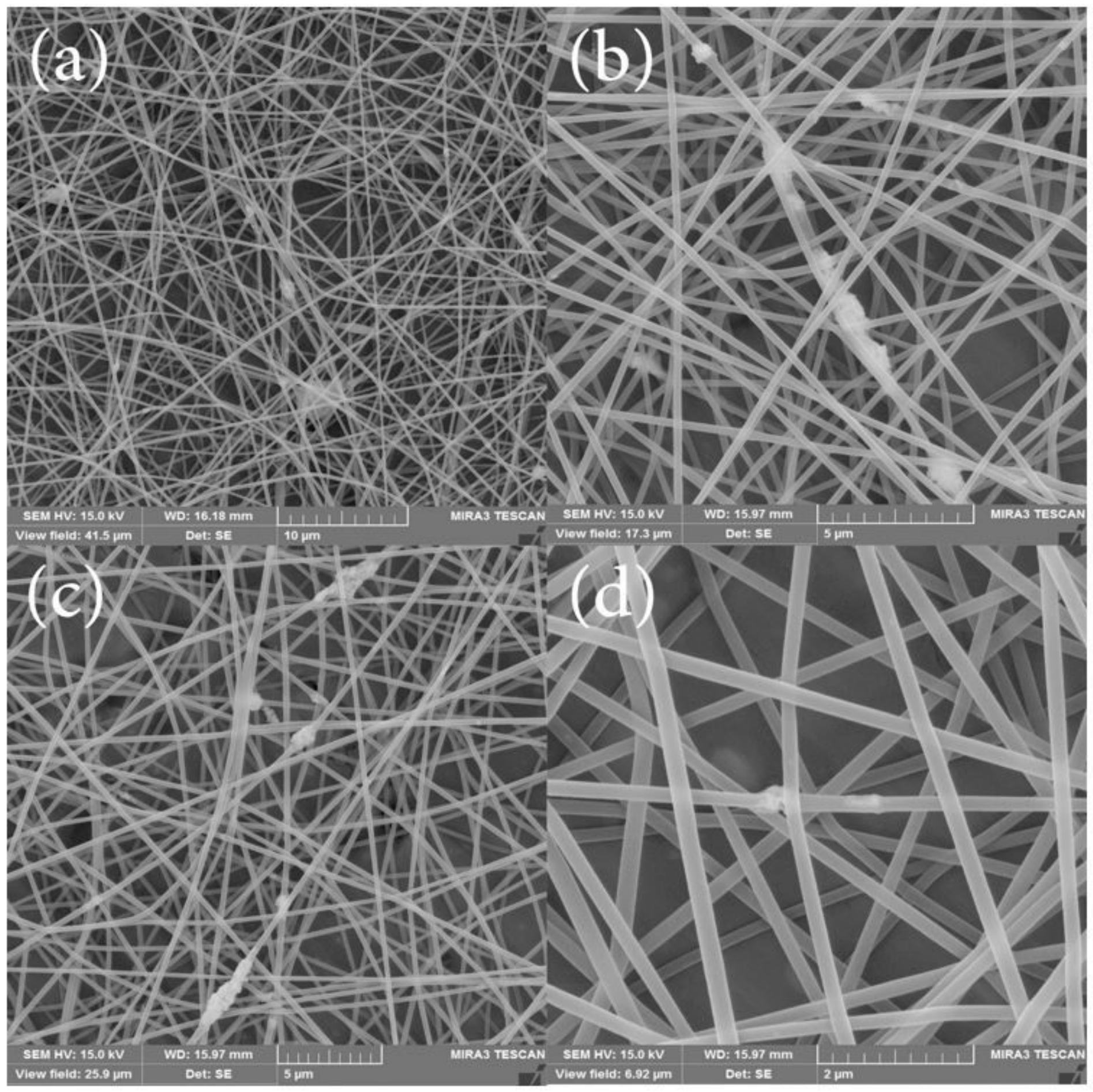

Figure 8

SEM images of Ag/PVA (10\%:90\%) nano-fibers 


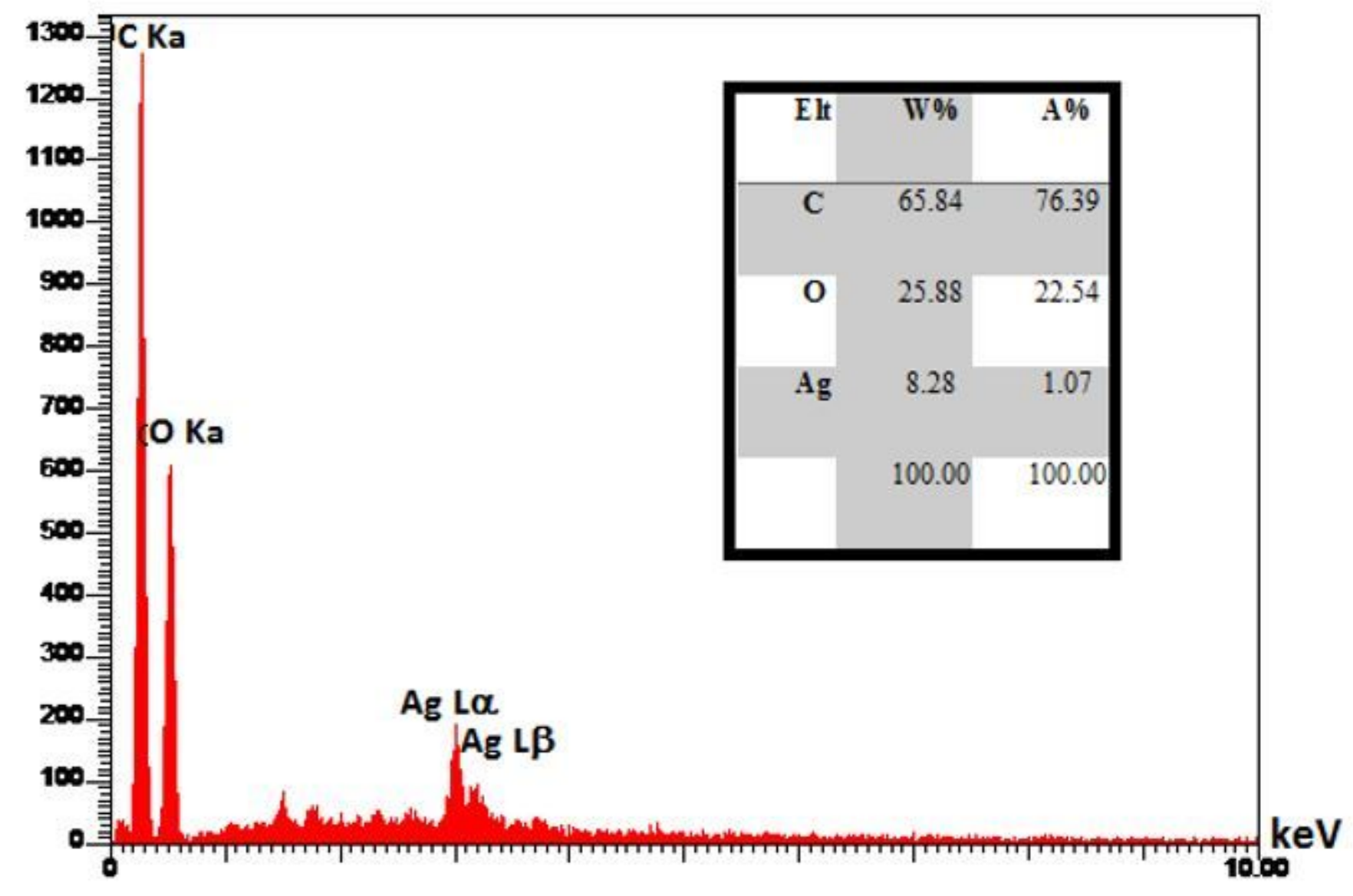

Figure 9

EDX spectra of Ag/PVA nano-fibers 


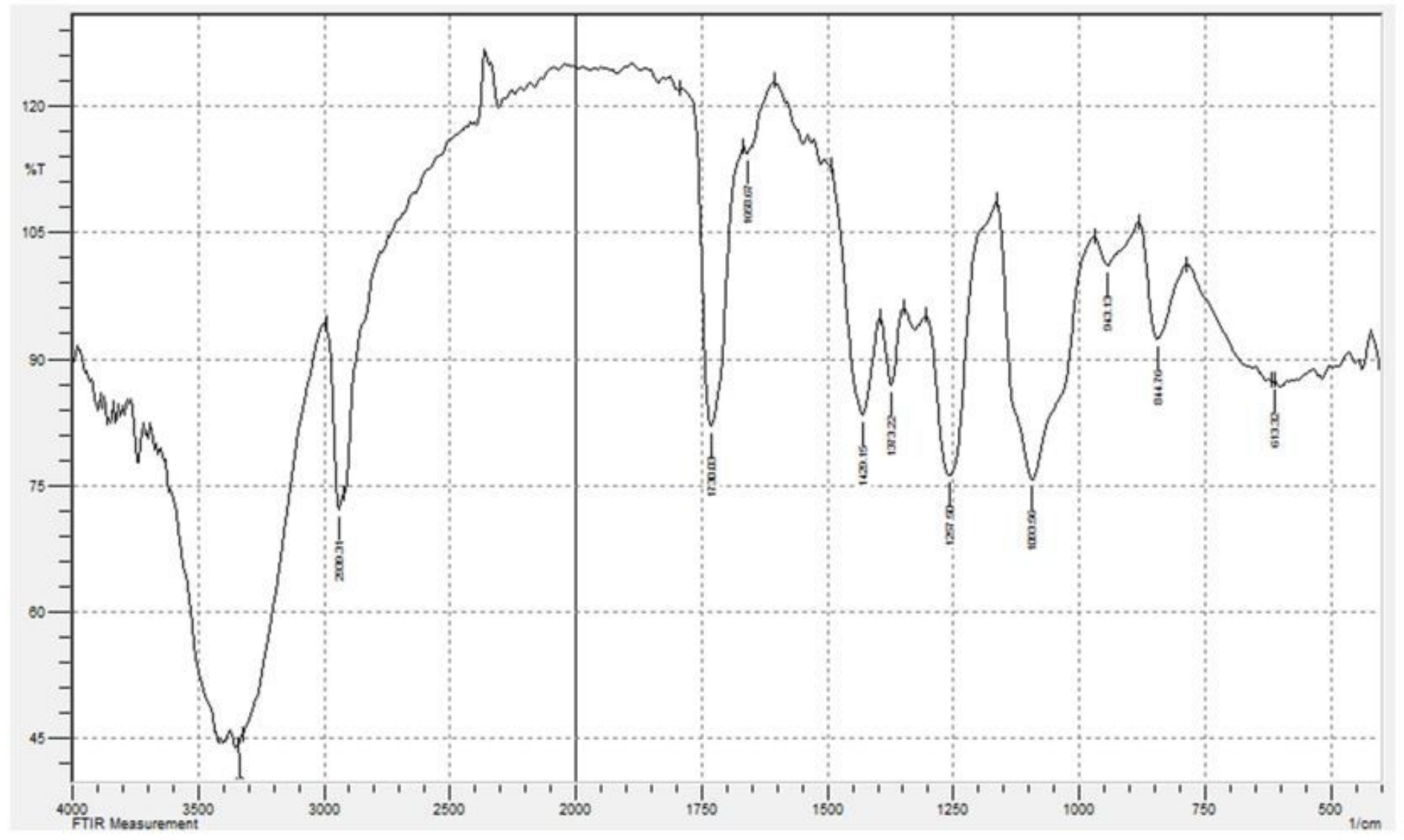

Figure 10

FT-IR spectrum of PVA nano-fibers 


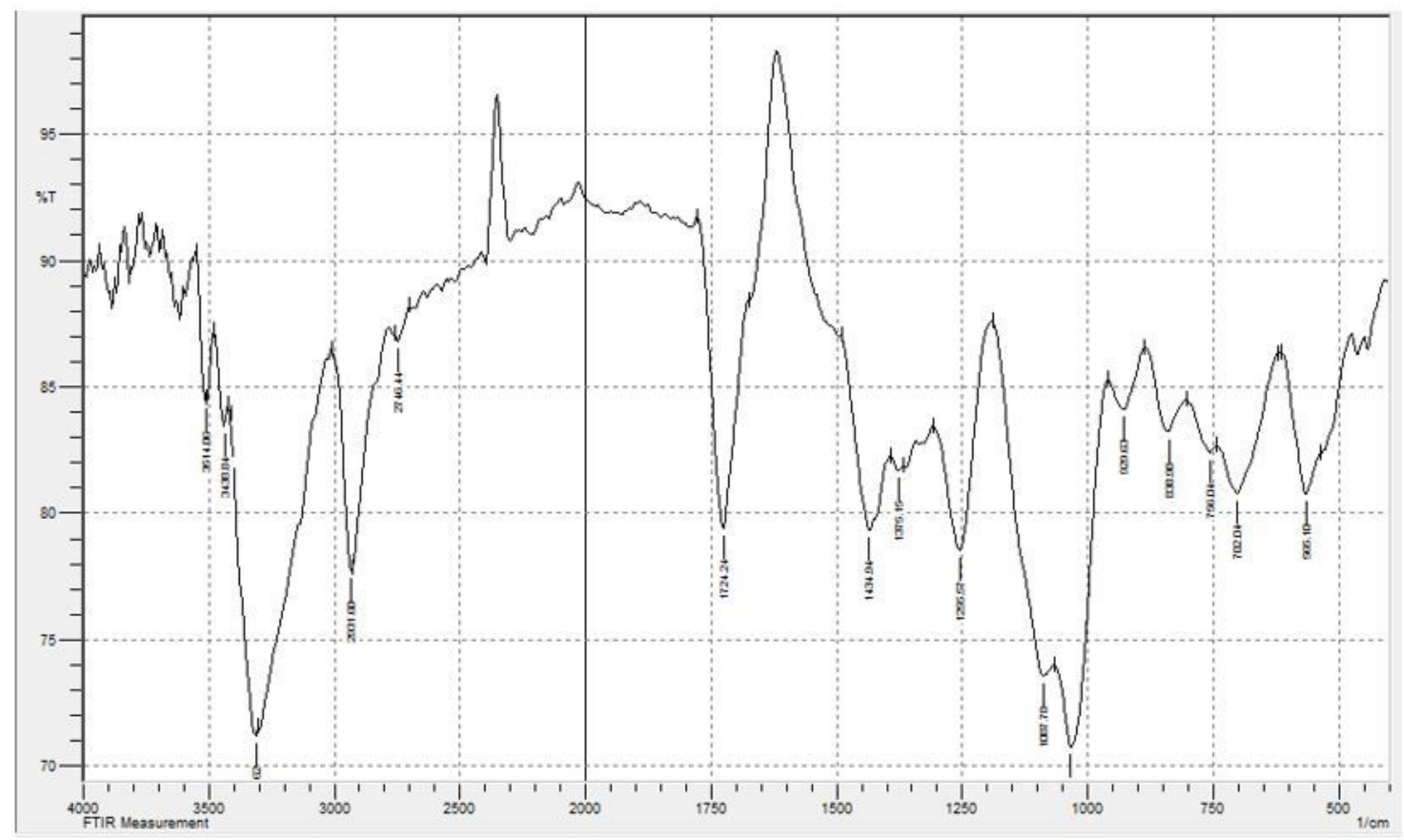

Figure 11

FT-IR spectra of Ag/PVA nano-fibers 


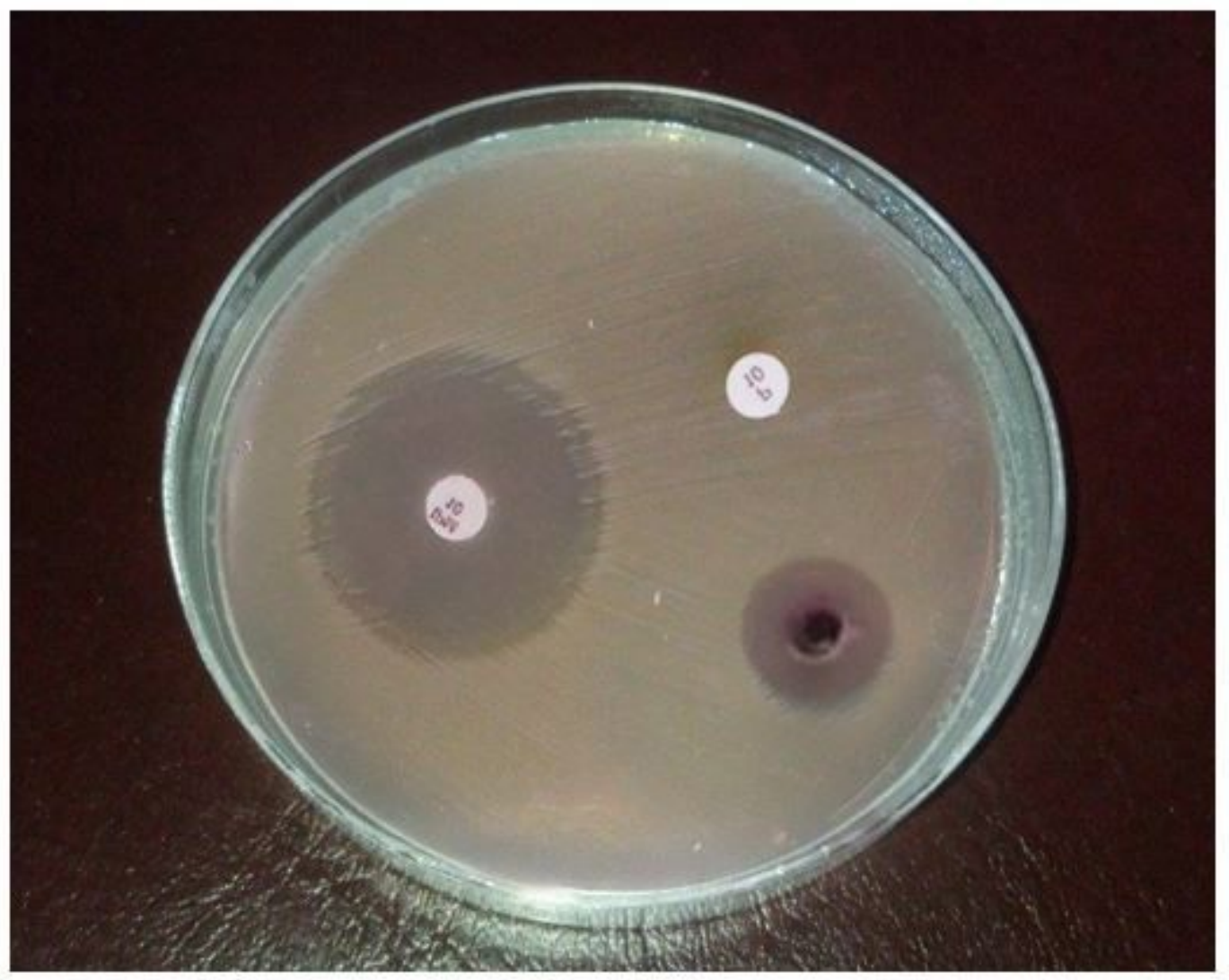

Figure 12

The antibacterial efficacy of Ag/PVA nano-fiber against E.Coli 


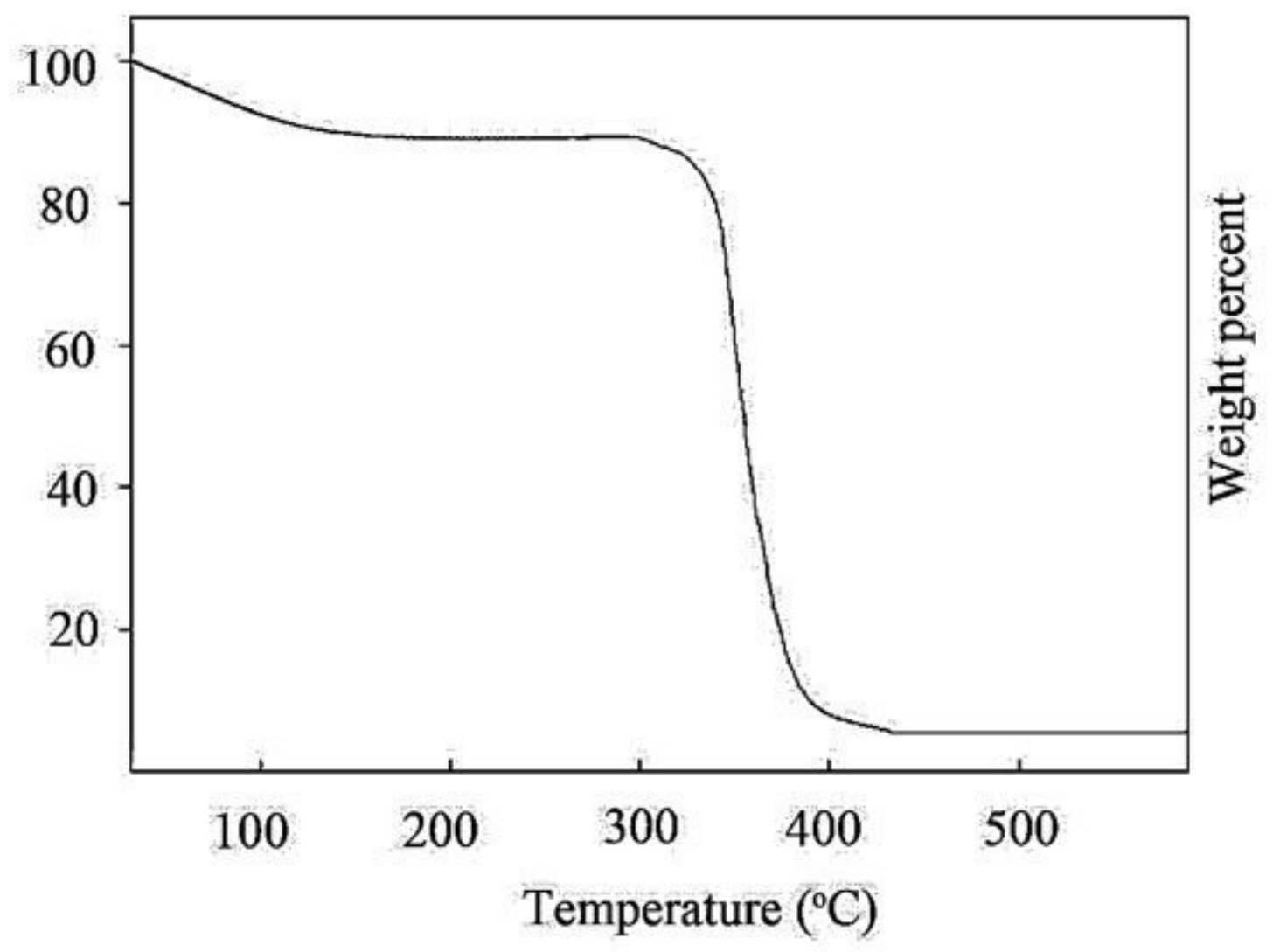

Figure 13

TGA graph of Ag/PVA nano-fibers 


\section{Char Effect}

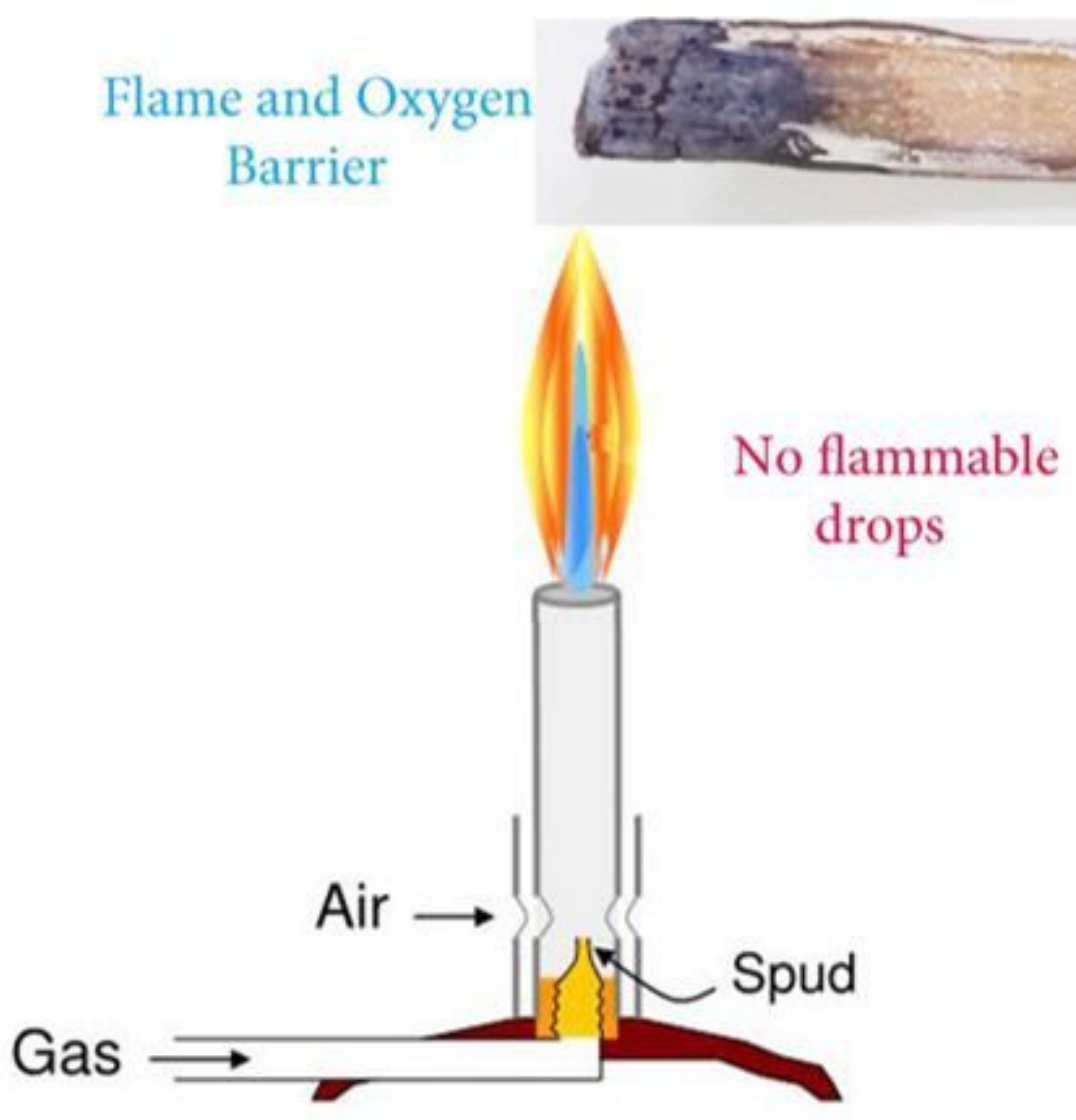

Figure 14

Schematic of UL-94 test of Ag/PVA nano-fibers 